\title{
CCD photometry and proper motions of late-type stars in the young open cluster Stock 2
}

\author{
D.C. Foster ${ }^{1}$, A. Theissen ${ }^{1}$, C.J. Butler ${ }^{1}$, W.R.J. Rolleston ${ }^{2}$, P.B. Byrne ${ }^{1}$, and S.L. Hawley ${ }^{3}$ \\ 1 Armagh Observatory, College Hill, Armagh BT61 9DG, N. Ireland \\ e-mail: ath@star.arm.ac.uk \\ 2 Department of Pure and Applied Physics, The Queen's University of Belfast, Belfast BT7 1NN, N. Ireland \\ 3 Department of Physics and Astronomy, Michigan State University, E. Lansing, Michigan 48824, U.S.A.
}

Received December 8, 1998; accepted February 8, 2000

\begin{abstract}
We present the results of the first CCD $B V R I$ photometric and proper motion study of late-type stars in the young open cluster Stock 2 .

Twenty-one fields of approximately $5^{\prime} \times 5^{\prime}$ size have been observed photometrically, from which we have identified 118 candidate members based on their positions on colour-magnitude diagrams relative to theoretical isochrones. From a comparison of the known star density of the Pleiades, we estimate the contamination of this selection process due to background stars to be as large as $\sim 50 \%$. However, only 22 of those 118 candidate members have proper motions consistent with membership, suggesting that the contamination is of the order of $80 \%$.

Additional candidate members were found by means of a proper motion analysis of Schmidt plate material for a $3^{\circ} \times 3^{\circ}$ field containing the cluster. The cluster proper motion allows us to separate members from background and foreground stars. We have found 634 stars with a membership probability $\geq 50 \%$ down to a limiting magnitude of $B \approx 20$, corresponding to late-M dwarfs at the distance of Stock $2^{1}$.
\end{abstract}

Key words: stars: evolution — stars: late-type — open clusters: individual; stock 2

\section{Introduction}

Stock 2 is an open cluster situated in the Orion spiral arm almost in front of the double cluster $\mathrm{h} \& \chi$ Persei.

Send offprint requests to: A. Theissen

1 Table 5 and the Table Appendix are only available in electronic form at the CDS via anonymous ftp to cdsarc.u-strasbg.fr (130.79.128.5) or via http://cdsweb.ustrasbg.fr/Abstract.html
The nominal cluster centre as given by Lynga (1987) is $\alpha(1950)=2^{\mathrm{h}} 11.4^{\mathrm{m}}, \delta(1950)=59^{\circ} 2^{\prime}\left(\right.$ or $l=133.4^{\circ}$, $\left.b=-1.9^{\circ}\right)$. It was discovered by Stock (1956) from a study of photographic plates taken at the Warner and Swasey Observatory, who originally estimated the distance of the cluster to be $\sim 300 \mathrm{pc}$, corresponding to a distance modulus of $m-M=7.5$. Results from $U B V$ photoelectric photometry by Krzemiński \& Serkowski (1967) agreed with this distance, and they found a mean reddening of $E_{B-V}=0.375$. More recently, Piskunov (1980), using existing photometry, redetermined the ages and distances of 68 open clusters. For Stock 2, he found the age to be $100 \mathrm{Myr}$, and the distance modulus to be $m-M=7.4$. In a study of the integrated parameters of open clusters, Pandey et al. (1989) also lists details of Stock 2, viz. a distance of $m-M=8.36$ and a reddening of $E_{B-V}=0.30$, using the age as determined by Piskunov. The large differences in distance moduli are due to a mixture of apparent and true distance modulus. In the most recent study on Stock 2, Robichon et al. (1997 and 1999) derived cluster membership of 5 stars using Hipparchos data, and concluded a cluster age of $170 \mathrm{Myr}$. We have chosen to adopt the closer cluster distance $(m-M=7.4)$. The faintest cluster member detected by these studies has a magnitude of $V \sim 12$. To date, there have been no studies of the lower mass cluster members.

This paper is the fourth in a series devoted to finding low mass cluster members in nearby $(<400 \mathrm{pc})$ open clusters. Previous papers discussed the open clusters NGC 5460 (Barrado \& Byrne 1995), IC 2602 (Foster et al. 1997) and IC 2391 (Rolleston \& Byrne 1997). As a consequence of the proximity of these open clusters, and the intrinsic faintness of late-type stars, it is difficult to separate cluster members from the field dwarfs and giants. To date, we have found member candidates by comparing CCD colour magnitude diagrams with theoretical isochrones. Radial velocities and spectral classification cannot be used 
Table 1. Coordinates of the field centres

\begin{tabular}{cccc}
\hline \multicolumn{2}{c}{$\alpha(\mathrm{J} 2000.0) \delta$} & \multicolumn{2}{c}{$\alpha(\mathrm{J} 2000.0) \delta$} \\
\hline $2: 14: 40$ & $59: 08: 28$ & $2: 14: 40$ & $59: 23: 28$ \\
$2: 15: 19$ & $59: 08: 28$ & $2: 15: 19$ & $59: 23: 28$ \\
$2: 14: 01$ & $59: 13: 28$ & $2: 15: 58$ & $59: 23: 28$ \\
$2: 14: 40$ & $59: 13: 28$ & $2: 14: 00$ & $59: 28: 28$ \\
$2: 15: 19$ & $59: 13: 28$ & $2: 15: 58$ & $59: 28: 28$ \\
$2: 15: 58$ & $59: 13: 28$ & $2: 13: 60$ & $59: 33: 28$ \\
$2: 14: 00$ & $59: 18: 28$ & $2: 14: 40$ & $59: 33: 28$ \\
$2: 14: 40$ & $59: 18: 28$ & $2: 15: 19$ & $59: 33: 28$ \\
$2: 15: 19$ & $59: 18: 28$ & $2: 15: 58$ & $59: 33: 28$ \\
$2: 15: 58$ & $59: 18: 28$ & $2: 14: 39$ & $59: 38: 28$ \\
$2: 14: 00$ & $59: 23: 28$ & & \\
\hline
\end{tabular}

as a first approach because of the large number of contaminating objects. X-ray studies have been used to identify cluster members (see Randich et al. 1995; Prosser et al. 1995). However, this method introduces a bias towards active stars (Jeffries \& Tolley 1998). In this paper, we demonstrate the effectiveness of combining proper motion data with CCD photometry to select cluster members. An established list of cluster members at the faint end of the main sequence is essential for studies of luminosity functions, the evolution of lithium abundance (e.g. Jeffries 1997), and the evolution of angular momentum (e.g. Stauffer et al. 1997).

Section 2 describes the CCD photometry and Sect. 3 the proper motion data and reduction. The method used for identifying cluster members is described in Sect. 4.

\section{CCD photometry}

\subsection{Observations}

CCD photometry was obtained using the JKT on La Palma on the nights of the 20th and 21st October 1995. A $1024 \times 1024$ pixel Tek CCD was used at the Cassegrain focus, which gives a pixel scale corresponding to 0.33 arcsec on the sky. Observations were made through Johnson $B V$ and Harris $R I$ filters. Exposure times for the cluster fields were typically 700:100:60:60 seconds respectively. The coordinates of the $215 \times 5$ arcmin fields are listed in Table 1 . Repeat observations of each field were sacrificed in favour of total spatial coverage because of the extremely long readout time of the data acquisition system used on the telescope at that time ( $\sim 5 \mathrm{~min})$. A range of photometric standards from Landolt (1992) were observed during the course of each night.

\subsection{Data reduction and calibration}

The basic CCD reduction was carried out using IRAF's CCDRED package (Tody 1986). The raw images were trimmed
Table 2. The mean photometric errors present in the Stock 2 photometry as a function of magnitude

\begin{tabular}{lclll}
\hline Mag. & $V$ & $B-V$ & $V-R$ & $R-I$ \\
\hline$V \leq 15$ & 0.01 & 0.01 & 0.01 & 0.02 \\
$15<V \leq 17$ & 0.01 & 0.01 & 0.01 & 0.01 \\
$17<V \leq 18$ & 0.01 & 0.02 & 0.02 & 0.02 \\
$18<V \leq 19$ & 0.02 & 0.03 & 0.03 & 0.03 \\
$19<V \leq 20$ & 0.04 & 0.07 & 0.06 & 0.04 \\
$V>20$ & 0.07 & 0.11 & 0.09 & 0.06 \\
\hline
\end{tabular}

and overscan subtracted. Multiple dome flatfields were averaged, and the data flatfielded using the resultant images. Dome flats were divided by sky flats to check that the dome was uniformly illuminated. No correction for such an effect was found necessary. The reduction of the crowded fields was carried out using the DAOPHOT (Stetson 1987; Stetson et al. 1990) package in IRAF. Cluster stars were analysed by means of point spread function (PSF) fitting. The stellar images were well sampled, and a gaussian PSF was applied. Standard star observations were reduced by means of aperture photometry, using an aperture of 14 arcsec in diameter, i.e. the same size as the one used by Landolt (1992).

We were not able to measure the extinction reliably, so we used mean values obtained from Derek Jones (private communication). Zero points and colour terms were derived in the standard way by applying linear transformation equations. For each star, errors in magnitude and colour were calculated. These include the statistical errors in flux, read-out noise of the CCD chip, and the errors in zero points and colour terms. The error calculations were performed within the IRAF tasks for photometry and fitting transformation equations (phot, fitparams, invertfit). Table 2 lists mean photometric errors, i.e. average values for several magnitude bins.

Using the STARLINK package ASTROM, about 10 to 20 stars on each CCD frame were manually identified on SuperCOSMOs scans of a POSSII photographic plate, and a transformation performed from pixel to equatorial coordinates. The resulting positions of all stars on the CCD frames are accurate to $\sim 0.1$ arcsec.

A complete list of all stars with coordinates and CCD photometry is available by anonymous ftp from the Centre de Données Stellaire, Strasbourg, or from the Armagh Observatory WWW server ${ }^{2}$.

\section{Proper motions}

\subsection{Data and reduction}

The proper motion survey of Stock 2 is based on Supercosmos scans (Hambly et al. 1998) of three POSS

\footnotetext{
${ }^{2} \mathrm{ftp}: / /$ www.arm.ac.uk/pub/ath/stock2/
} 
Table 3. Plate Material for proper motion study of Stock 2

\begin{tabular}{lccclll}
\hline Plate Id. & $\alpha$ & $\delta$ & Epoch & Emulsion & Filter & Medium \\
\hline POSSI E597 & $2: 19$ & $60: 02$ & 1952.708 & 103AE & 2444 & Glass Copy \\
POSSII R114 & $1: 54$ & $60: 00$ & 1989.965 & IIIaF & RG 630 & Film Copy \\
POSSII B114 & $1: 54$ & $60: 00$ & 1987.744 & IIIaJ & GG 395 & Film Copy \\
\hline
\end{tabular}

plates listed in Table 3 . A region of $3^{\circ} \times 3^{\circ}$ has been scanned about the nominal cluster centre with a positional accuracy of $\sim 1 \mu \mathrm{m}$. The scans were performed in the so-called Image Analysis Mode (Beard et al. 1990), which automatically deblends objects and rejects extended objects like galaxies. A transformation from plate to equatorial coordinates in the FK5 system has been performed using PPM stars on the plate. Using software provided by the Royal Observatory of Edinburgh, the following reduction steps were performed:

The first task is to pair up the objects on the first and second epoch plate (E597 and R114, respectively). This involves identifying common objects, determining and applying a transformation from the coordinate system of the first epoch plate to the second epoch plate, and pairing up objects that lie at the same position (allowing for some small margin of error).

1000 bright stars in common are used to fit and apply a linear coordinate transformation of the form

$x_{\mathrm{e}}=a+b x_{\mathrm{m}}+c y_{\mathrm{m}}$

$y_{\mathrm{e}}=d+e x_{\mathrm{m}}+f y_{\mathrm{m}}$

to the plates. Here $x$ and $y$ refers to the plate coordinates, subscript $e$ denotes the expected coordinate from the second epoch plate, and subscript $m$ the measured coordinate on the first epoch plate. A transformation of this form will correct for differences in plate origin, scale, orientation, shear and squash. Including shear and squash did not improve our fit, so we constrained the fit by $b=-f$ and $c=e$. The root mean square error of the fit was $5 \mu \mathrm{m}$ which corresponds to 0.34 arcsec.

Stars with equal coordinates on both plates (i.e. the nearest star within $50 \mu \mathrm{m}$ ) were considered as identical, and relative proper motions were derived. However, two more systematic effects have to be accounted for.

Distortion of the plate emulsion can have serious effects on the position of stars when large areas of the plate are analysed. To avoid having to fit these complicated distortions, the plates were subdivided into smaller regions $(12 \times 12=144$ boxes $)$, and proper motions determined independently within each region. Secondly, stellar positions are magnitude dependent due to the non-linearity of the plate emulsions.

The plate coordinate transformation was re-applied to stars of the first epoch plate in each of the 144 sub-regions independently. This was done in the same way as described above. Reference stars to fit the transformation equations were chosen automatically within a suitable magnitude range to avoid too faint or too bright and saturated stars. The residual positions were fitted as a function of magnitude and the residuals of this fit subtracted from the first epoch plate positions. This fit was re-iterated, until the fit converged. The remaining positional differences are the actual proper motions.

Within each of the 144 subregions, a background field distribution is visible in vector point diagrams (VPDs), centered around zero proper motion. More precisely, the average centre coordinate of the proper motion distributions of all 144 subregions is $(-0.09,0.37) \pm(0.60,0.37)$ arcsec per year. Each subfield has thus the same zero point in proper motion, and the results of all subregions are merged to one VPD in Fig. 3.

\subsection{Plate photometry}

The method of separating cluster members from background objects by means of a proper motion analysis is to identify a group of common proper motion stars that are distinct from the background population. However, plotting a VPD of the stellar proper motions at this stage would reveal very little, as the cluster stars are masked by the overwhelming majority of some $10^{5}$ background objects. It is first necessary to preselect background stars by means of a colour magnitude diagram of the plate photometry, calibrated using the new CCD photometry.

Since our plate material supplies magnitudes in the photographic system, the CCD photometry has first to be transformed to the natural photographic system using the equations

$$
\begin{aligned}
B-B_{\mathrm{J}}= & (0.32 \pm 0.03)(B-V) \\
R-R_{63 \mathrm{~F}}= & 0.013+0.204(R-I)-0.100(R-I)^{2} \\
& -0.0295(R-I)^{3}
\end{aligned}
$$

from Blair \& Filmore (1982), and Bessell (1986) respectively, where $B_{\mathrm{J}}$ and $R_{63 \mathrm{~F}}$ denote magnitudes in the photographic system and $B V R I$ are CCD measured magnitudes in the standard system. A cubic spline function was fitted to transform CCD $B V R I$ magnitudes to photographic $B_{\mathrm{J}}$ and $R_{63 \mathrm{~F}}$ magnitudes; points for this fit were chosen interactively so as to allow for some extrapolation beyond the bright and faint limit of the CCD photometry. Finally, the fit was applied to all stars on the plate. The residuals of the fit of naturalized CCD and calibrated plate photometry have a scatter of $\approx 0.15$ magnitudes both for $B_{\mathrm{J}}$ and $R_{63 \mathrm{~F}}$. 
Unfortunately, the CCD photometry covers only a small central region of the scanned plate. Further investigation suggested that there was a position dependent colour-shift across the plate. If colour-magnitude diagrams were plotted for different sub-regions of the plate, the position of the density distribution of background field stars in the colour-magnitude plane varied by up to 0.1 magnitudes in colour. Whether this is due to problems with the photometric calibration or the effect of differential reddening across the field (due to the low Galactic latitude, $b \sim 1.9^{\circ}$, of the cluster) is unclear. The shifts are consistent with an increased reddening towards the Galactic plane, but also with a colour-effect towards the edge of the plate. In the absence of more photometry with which to calibrate the photographic data, the plate material was used with the calibration determined from the central region.

\section{Selecting cluster member candidates}

\subsection{Considering only CCD photometry}

Following the same procedure as in Foster et al. (1997) and Rolleston \& Byrne (1997), candidate cluster members were selected using the theoretical pre-main sequence isochrones of D'Antona \& Mazzitelli (1994), and in particular those resulting from computations using opacities from Alexander et al. (1989) and Rodgers \& Iglesias (1992) and the mixing model of Canuto \& Mazzitelli (1991).

Isochrones are presented in terms of stellar effective temperature and luminosity, and need to be transformed to the observational domain of magnitude and colour. Unfortunately, these calibrations are not yet well defined for the M-type dwarfs. Stauffer et al. (1995) have made a comparison of the magnitudes and colours of known Pleiads with combinations of isochrones and transformations, viz. models provided by Vandenberg and Swenson (private communication) in addition to those of D'Antona \& Mazzitelli (1994). They found that the best agreement between the theoretical tracks and the observed photometry was achieved using the D'Antona \& Mazzitelli models mentioned above, combined with an ad hoc "tuned" temperature scale. However, of the observationally defined transformations, the temperature scales from Kirkpatrick et al. (1993) with bolometric corrections from Bessell (1991) provided the closest fit to the data. Thus, we have adopted the temperature scales from Kirkpatrick et al. and bolometric corrections from a more recent paper by Bessell (1995) for the range $T_{\text {eff }} \leq 3500 \mathrm{~K}$, and the temperature scale of Bessell (1991) for $4000 \mathrm{~K} \geq T_{\text {eff }}>3500 \mathrm{~K}$. For $T_{\text {eff }}>4000 \mathrm{~K}$, we have used the temperature scales and bolometric corrections by Wood \& Bessell (private communication) which are available via anonymous ftp from mso.anu.edu.au.

The selection regions for cluster membership in the $B, B-V$ and $R, R-I$ colour-magnitude diagrams were initially defined by the 80 and 120 Myr isochrones. This region was subsequently broadened to allow for an uncertainty of $0.2 \mathrm{mag}$ in the distance modulus (Stock 1956; Piskunov 1980), an uncertainty of $0.01 \mathrm{mag}$ in the reddening (Krzemiński \& Serkowski 1967), the photometric errors as listed in Table 2, and the effects of binarity. In the case of reddening, only the redder isochrone, i.e. the $80 \mathrm{Myr}$ one, is shifted redwards, the bluer one not. The effect of binarity on the location of stars with respect to the isochrones depends on the frequency of binaries and the distribution of their mass ratios. However, Dabrowski \& Beardsley (1977) have shown that the maximum increase in brightness would correspond to $\sim 0.8$ magnitudes, and hence this has been incorporated in our bright selection limit.

Only stars fullfilling the selection criteria in both colour-magnitude diagrams were considered as candidate cluster members, and 118 stars with magnitudes in the range $14.2<V<20.4$ were identified as such. The colourmagnitude diagrams are shown in Figs. 1 and 2.

In our previous paper, Foster et al. (1997) estimated the contamination due to background stars for IC 2602 using CCD observations of an offset field, and as a second approach, by comparison with previously determined star densities for the Pleiades. No offset frame has been observed in the case of Stock 2, and only the latter method can be applied here. This comparison is justified given the similar age and same richness class (Lynga 1987) of Stock 2 and the Pleiades. In an equivalent area imaged by our CCD frames, one would expect to find 52 Pleiads. Hence, our list of CCD photometrically selected candidate members may be contaminated by background stars by up to $50 \%$.

\subsection{Considering proper motion data}

The first step in the analysis was to remove as many of the field stars as possible from consideration, using the plate photometry. A photometric selection was made from the photographic $B, B-R$ colour-magnitude diagram using a 100 Myr isochrone from D'Antona \& Mazzitelli (1994), using the temperature scale and bolometric corrections as described above, and including the transformation to the photographic photometry system as described in Sect. 3.1. Stars lying within a band 0.8 magnitudes fainter and 1.8 magnitudes brighter were selected as possible cluster members. This reduced the number of stars to be considered from more than 300000 to $\sim 7500$. The band around the isochrone was chosen rather broad because of the errors in plate photometry calibration and the position dependent colour-shift mentioned in Sect. 3.2. Using a $170 \mathrm{Myr}$ isochrone instead of $100 \mathrm{Myr}$, thus following the age determination of Robichon et al. (1997 and 1999), would not change the result in a significant way.

The proper motions of the selected stars were analysed using the method described by Sanders (1971). 

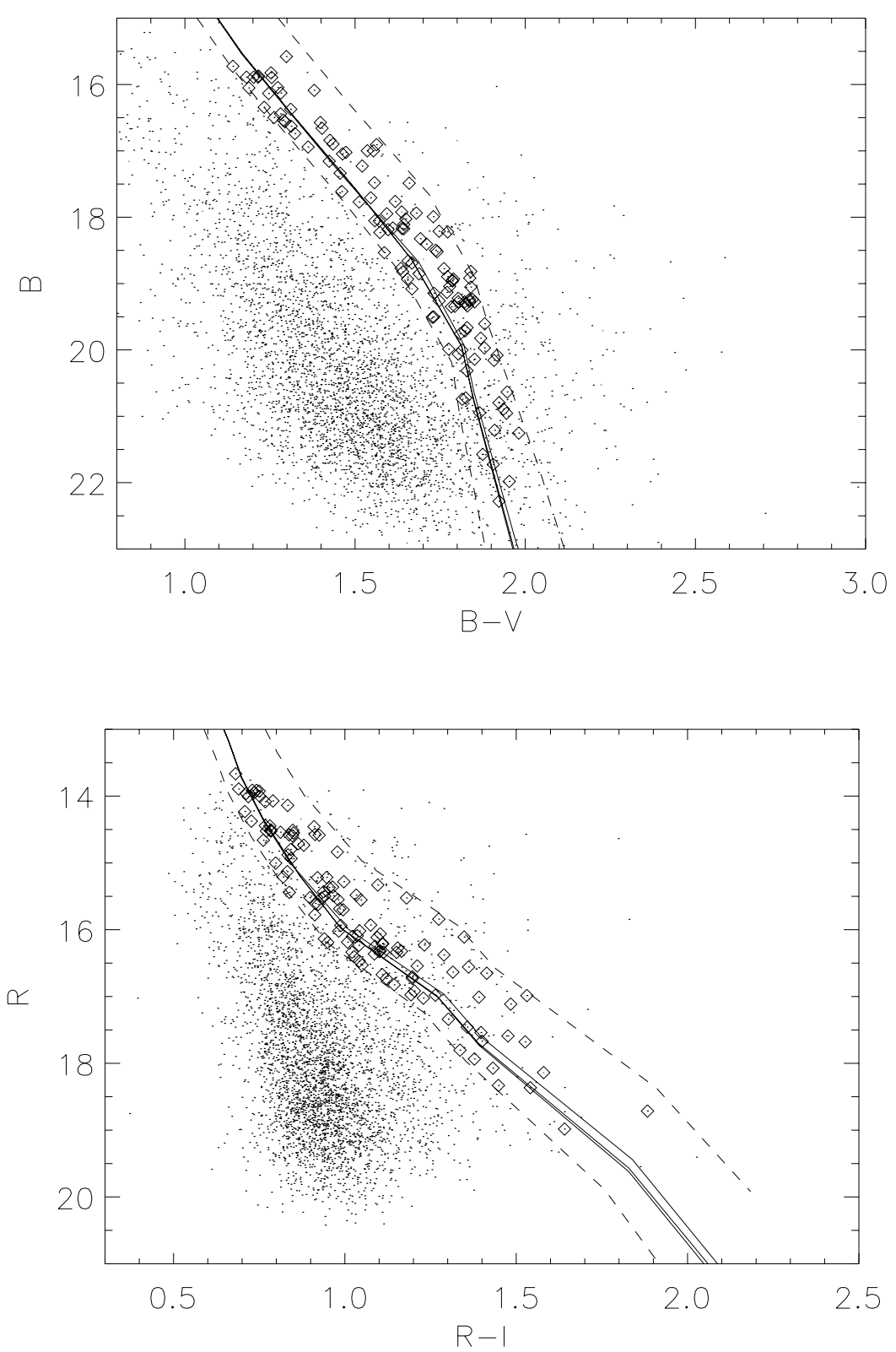

Fig. 1. $B$ vs. $B-V$ colour-magnitude diagram from CCD photometry. The solid lines represent isochrones for cluster ages of $80,100,120$, and 200 Myr, respectively. The region between the dashed lines defines the location of possible cluster members, as described in detail in Sect. 4.1. Diamonds in both colour-magnitude diagrams indicate cluster member candidates
The distribution of stars in the VPD is modeled as the sum of two bivariate Gaussians, a circular distribution for the cluster stars and an elliptical distribution for the field stars:

$\rho\left(\mu_{x_{i}}, \mu_{y_{i}}\right)=\phi_{\mathrm{c}}\left(\mu_{x_{i}}, \mu_{y_{i}}\right)+\phi_{\mathrm{f}}\left(\mu_{x_{i}}, \mu_{y_{i}}\right)$

where

$$
\begin{aligned}
\phi_{\mathrm{f}}= & \frac{f N}{2 \pi \Sigma_{x} \Sigma_{y}} \\
& \exp \left\{-\frac{1}{2}\left[\left(\frac{\mu_{x_{i}}-\mu_{x_{\mathrm{f}}}}{\Sigma_{x}}\right)^{2}+\left(\frac{\mu_{y_{i}}-\mu_{y_{\mathrm{f}}}}{\Sigma_{y}}\right)^{2}\right]\right\}
\end{aligned}
$$

is the field star distribution,

$\phi_{\mathrm{c}}=\frac{(1-f) N}{2 \pi \sigma^{2}}$
Fig. 2. Same as Fig. 1, but showing the $R$ vs. $R-I$ colour-magnitude diagram

$$
\exp \left\{-\frac{1}{2}\left[\left(\frac{\mu_{x_{i}}-\mu_{x_{\mathrm{c}}}}{\sigma}\right)^{2}+\left(\frac{\mu_{y_{i}}-\mu_{y_{\mathrm{c}}}}{\sigma}\right)^{2}\right]\right\}
$$

the cluster star distribution, $N$ is the total number of stars in the model, $f$ is the fraction of field stars, $\Sigma_{x} \& \Sigma_{y}$ are the standard distribution of the field star positions in $x$ and $y, \sigma$ is the standard deviation of the cluster population, $\left(\mu_{x_{\mathrm{f}}}, \mu_{y_{\mathrm{f}}}\right) \&\left(\mu_{x_{\mathrm{c}}}, \mu_{y_{\mathrm{c}}}\right)$ the field and cluster centres in the VPD, and $\left(\mu_{x_{i}}, \mu_{y_{i}}\right)$ the proper motion of the $i$ th star in the fit. Thus, there were a total of 8 parameters to be determined. The model as formulated above contains no dependence on the spatial distribution of cluster and field stars. In their proper motion studies of Praesepe, Jones \& Stauffer (1991) and Hambly et al. (1995) use a model with a uniform surface density of field stars, and a radial 
Table 4. The final solutions to the proper-motion distribution model by magnitude bin

\begin{tabular}{llllllllll}
\hline Bin & $f$ & $\mu_{x_{\mathrm{c}}}$ & \multicolumn{1}{c}{$\mu_{y_{\mathrm{c}}}$} & \multicolumn{1}{c}{$\mu_{x_{\mathrm{f}}}$} & \multicolumn{1}{c}{$\mu_{y_{\mathrm{f}}}$} & $\sigma$ & $\Sigma_{x}$ & $\Sigma_{y}$ & $N$ \\
\hline $12<B \leq 14$ & 0.875 & 17.140 & -15.534 & -0.017 & -1.143 & 4.531 & 9.441 & 7.740 & 160 \\
$14<B \leq 16$ & 0.922 & 16.752 & -12.951 & 0.208 & -1.078 & 4.651 & 7.424 & 6.793 & 1306 \\
$16<B \leq 18$ & 0.916 & 15.216 & -12.876 & -0.245 & -0.720 & 5.449 & 6.136 & 5.690 & 2241 \\
$18<B \leq 20$ & 0.872 & 14.600 & -9.263 & -0.675 & -0.302 & 6.663 & 6.323 & 5.954 & 1756 \\
\hline
\end{tabular}

exponential decrease in cluster members (the characteristic radius being another free parameter) from the cluster centre. Their formulation is unsuitable for fitting the central regions of the cluster, as they were primarily interested in searching for members in the outer regions of Praesepe. We felt that an a priori assumption of the spatial distribution would discriminate against cluster members deviating from such a form and that the spatial aspect of the cluster distribution would be better analysed after a selection on the basis of proper motion.

The solution was determined iteratively using the method of maximum likelihood (see Sanders 1971 for further details), and employing the method of bisection (Press et al. 1992). Our model was adapted from a code written by Hambly (private communication), to solve for the root of each equation in turn until all equations could be satisfied with a single set of the 8 parameters. The code was found to be insensitive to the initial estimates of the parameters, but somewhat sensitive to the adopted data points. Points lying far from the centre of the VPD caused the code to either converge on an unrealistic model of the data containing a large fraction $(\sim 50 \%)$ of cluster members located close to the centre of the VPD, or crash as the result of a division-by-zero error (resulting from the extremely low density of field stars located far from the centre of the distribution). Thus for the purposes of determining the model, the data were restricted to stars with proper motions in the range $\left|\mu_{x}, \mu_{y}\right| \leq 30 \mathrm{mas} / \mathrm{yr}$. The model parameters were independently determined for each of the 4 magnitude bins spanning the range $12 \leq B \leq 20$, and the fitted parameters are shown in Table 4 .

Membership probabilities were calculated for each star based on the model using the ratio of the densities of cluster stars $(\phi)$ to cluster plus field stars $(\rho)$ :

$P\left(\mu_{x_{i}}, \mu_{y_{i}}\right)=\frac{\phi_{\mathrm{c}}\left(\mu_{x_{i}}, \mu_{y_{i}}\right)}{\rho\left(\mu_{x_{i}}, \mu_{y_{i}}\right)}$.

Stars with membership probabilities greater than $50 \%$ were considered candidate cluster members (634 in total) and are listed in Table 5.

A VPD is shown in Fig. 3 in which the cluster is clearly visible. At this stage, some shortcomings of the method in selecting cluster members become evident, especially in cases like ours where the contamination of the sample with background field stars is very high. In this context, it is interesting to note from Table 4 that the width of the Gaussian distribution fitting the cluster stars $(\sigma)$ increases with increasing magnitude. We believe this to be
Table 5. Coordinates, photographic photometry and proper motions of 634 Stock 2 stars with a membership probability $\geq 50 \%$ based on the proper motion analysis, ordered by magnitude. Only the first 10 stars are shown here. The complete table is available by anonymous ftp from the Centre de Données Stellaire, Strasbourg, or from the Armagh Observatory WWW server (ftp://www.arm.ac.uk/pub/ath/stock2/)

\begin{tabular}{|c|c|c|c|c|c|}
\hline \multicolumn{2}{|c|}{$\alpha(\mathrm{J} 2000.0) \delta$} & $B$ & $B-R$ & $\mu_{x}$ & $\mu_{y}$ \\
\hline $2: 18: 57.63$ & $58: 59: 50.4$ & 12.84 & 1.25 & 28.6 & -20.5 \\
\hline $2: 16: 00.47$ & $59: 28: 31.2$ & 13.16 & 1.12 & 13.0 & -13.8 \\
\hline $2: 18: 29.65$ & $60: 29: 59.7$ & 13.22 & 1.25 & 25.0 & -17.9 \\
\hline $2: 13: 44.80$ & 59:00:50.7 & 13.31 & 1.10 & 13.8 & -24.3 \\
\hline $2: 14: 34.65$ & $59: 29: 10.3$ & 13.31 & 1.34 & 26.2 & -24.4 \\
\hline $2: 19: 22.58$ & $60: 21: 34.1$ & 13.36 & 1.26 & 32.3 & -17.0 \\
\hline $2: 17: 14.74$ & $59: 31: 34.5$ & 13.40 & 1.33 & 22.9 & -13.1 \\
\hline $2: 18: 00.67$ & $60: 21: 11.8$ & 13.42 & 1.38 & 23.1 & -35.2 \\
\hline $2: 15: 08.48$ & 59:39:16.1 & 13.44 & 1.21 & 18.0 & -9.8 \\
\hline $2: 16: 33.93$ & $59: 24: 38.2$ & 13.55 & 1.16 & 25.0 & -24.5 \\
\hline
\end{tabular}

for the following reason. With increasing magnitude, stellar images on the plates become less distinct, and their positions, measured as intensity weighted centroids, become less accurate. As a consequence, proper motions for faint stars also become less accurate. This results in a wider distribution in the VPD. The fitting procedure, however, tends to regard all stars in the outer region in the VPD which are closer to the cluster centre (in our case stars in the lower right region of the VPD) as cluster members. This also has the effect that the width of the field star distribution $\left(\Sigma_{x}\right.$ and $\left.\Sigma_{y}\right)$ does not increase with increasing magnitude. Another consequence is that in the combined VPD in Fig. 3, where all magnitudes are plotted together, some non-members appear to be surrounded by members in regions away from the centre of the cluster distribution. Those non-members are brighter stars for which the cluster distribution is narrower than for the faint stars.

Consequently, the process of determining membership probabilities can lead to some stars having high membership probabilities, yet lying quite far from the centre of the cluster centre in the VPD. For example, the star at $(-5.8,-34.7)$ in Fig. 3 has a formal membership probability of $95 \%$, but is extremely unlikely to be a cluster member.

The sharp cut-off dividing the "candidate members" from the "non-members" is another consequence of our 


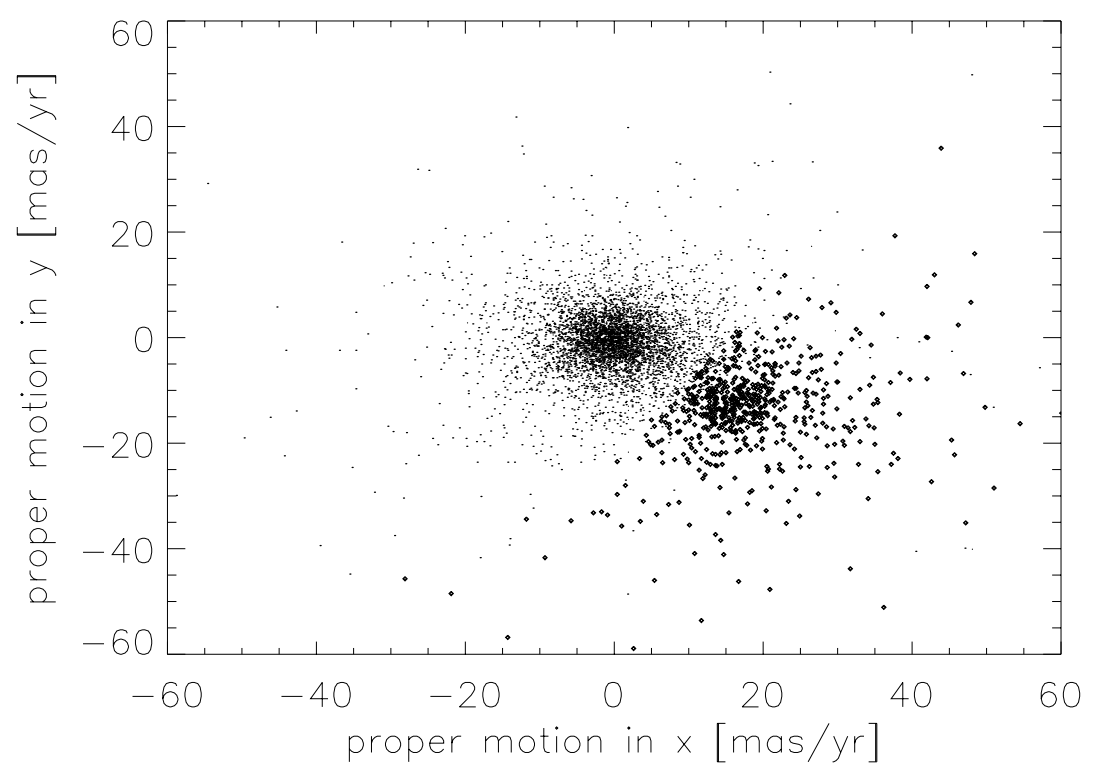

Fig. 3. Vector-point diagram after photometric preselection. Heavy dots denote stars with a membership probability greater than $50 \%$. The cluster is clearly separated from the background field stars. However, formal cluster members in the outer region of the distribution may not be members due to some shortcomings in the general method. See text for details. Note also that coordinates are plate coordinates transformed into milliarcseconds, but not right ascension and declination method used for determining membership probabilities. Considering Fig. 3 and Eq. (8), stars toward the lower right region of the VPD tend to be members because of divisions by small values of $\rho$, and the sharp cut-off represents the boundary where the membership probability becomes less than $50 \%$ due to divisions by high values of $\rho$. The list of stars with membership probabilities will probably contain some non-members and the level of this contamination has been estimated by applying the fitted distribution to "altered" datasets. The fit for the real dataset of $\left(\mu_{x}, \mu_{y}\right)$ was applied to the three "fake" datasets $\left(-\mu_{x}, \mu_{y}\right),\left(-\mu_{x},-\mu_{y}\right)$ and $\left(\mu_{x},-\mu_{y}\right)$, and the number of stars with membership probabilities greater than $50 \%$ was determined in each case. These numbers are shown in Table 6 , along with the estimated percentage of field stars calculated from the mean of the three "fake" selections. The number of stars in each bin, $N_{\mathrm{b}}$, is slightly larger than that used to determine the model, $N$, since no restriction on the stellar proper motions was imposed. For comparison, if no photometric pre-selection was made, the contamination in the $16<B \leq 18$ bin has been estimated to be greater than $75 \%$. This clearly demonstrates the importance of the combined approach used here. The list of non-members will certainly contain stars that are cluster members, but whose position in the VPD places them too close to the central distribution for them to have a membership probability greater than $50 \%$.

Thus, when compiling a list of stars, a final membership list must take into account the location of the star in the proper motion VPD as well as its membership probability.

This becomes evident once more when we plot the colour-magnitude diagram of the plate photometry (Fig. 4). The cluster members are highlighted therein as heavy dots. Given the estimated error in the photometry of $\approx 0.15 \mathrm{mag}$ both in $B_{\mathrm{J}}$ and $R_{63 \mathrm{~F}}$, we cannot expect the cluster members to lie nicely on an isochrone. In order to reduce these discrepancies, it would be desireable to obtain more extensive CCD photometry covering a larger area, and to correct for the position dependent effect mentioned in Sect. 3.2. Most notably, however, the diagram suggests that pushing the faint border used to preselect member candidates (lower dotted line in Fig. 4) to an even fainter limit will result in a selection of more cluster members. However, this is merely the result of increasing contamination of the sample with field stars. The field star distribution in the VPD soon becomes broader and grows so high that it dominates the total distribution. The large majority of field stars then makes it impossible to fit a bivariate Gaussian, which reduces the effectiveness of this method for distinguishing cluster members.

In summary, our analysis has certainly freed the sample from most of the field stars. However, if one wants to obtain a sample more free of field stars, spectroscopic methods have to be used to classify spectral types and distinguish background giants from cluster stars, or to identify the cluster stars as a group with different radial velocities than the field stars.

\subsection{Spatial distribution of candidate members}

The spatial distribution of the photometrically preselected stars along with the subset that have membership probabilities greater than $50 \%$ are shown in Fig. 5. In the upper two panels of this figure, only stars with a membership probability of $50 \%$ and more are plotted, and stars with 

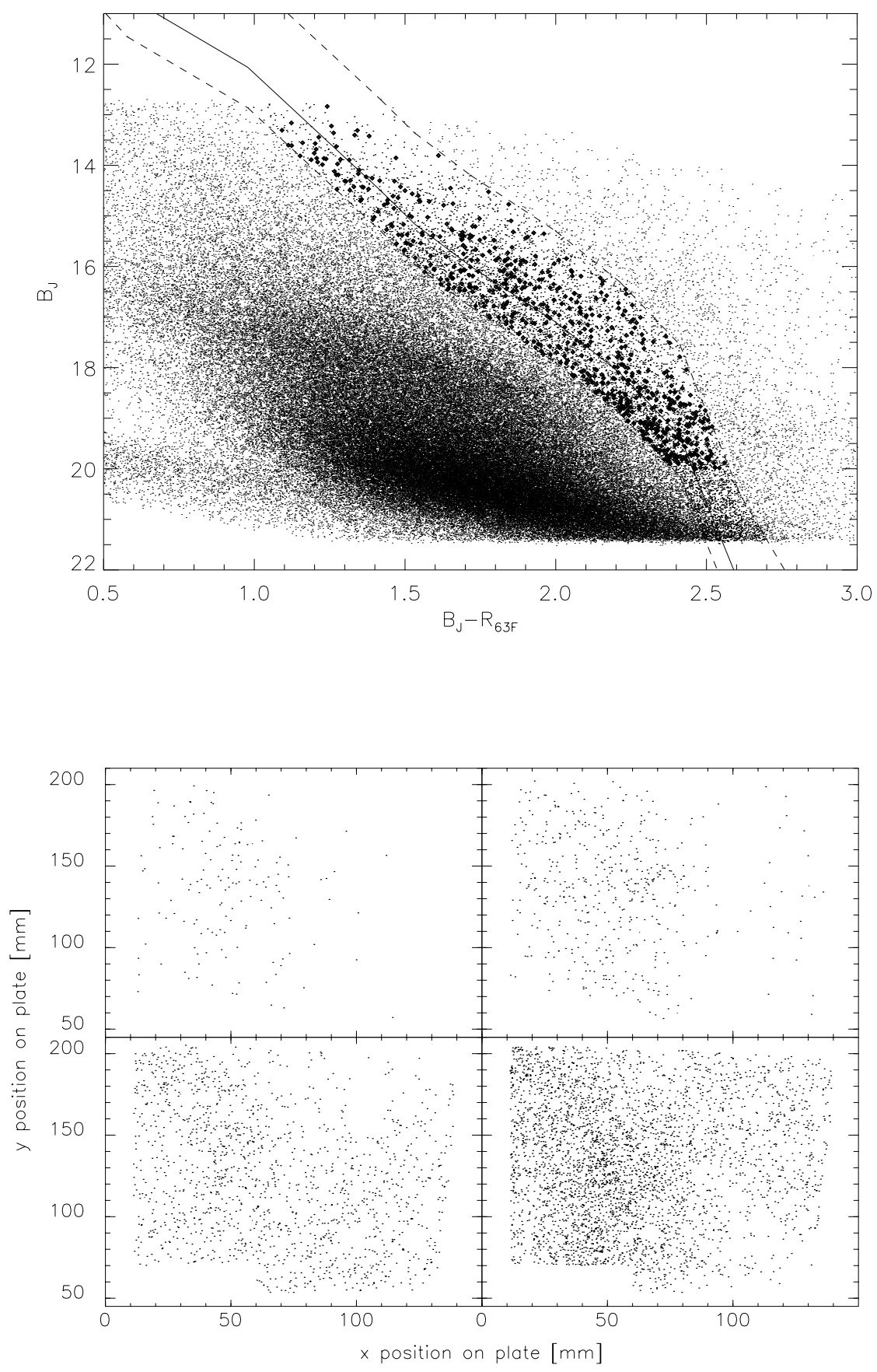

Fig. 4. Photographic colour-magnitude diagram of all stars. Stars with a membership probability greater than $50 \%$ are plotted as heavy dots. The solid line denotes the $100 \mathrm{Myr}$ isochrone, the dashed lines enclose the area out of which cluster member candidates have been preselected (see text for details, Sect. 4.2)

Fig. 5. The spatial positions of the stars. Upper left: membership probability $P \geq 50 \%$ and photographic magnitude $B \leq 16$; upper right: $P \geq 50 \%$ and $B>16$; lower left $P<50 \%$ and $B \leq 16$; lower right: $P<50 \%$ and $B>16$

Table 6. The number of stars with membership probabilities greater than $50 \%$ for the dataset (Col. 3 ) and the three "fake" datasets (Cols. 4-6) used in extimating the contamination due to background objects

\begin{tabular}{lllllll}
\hline Mag. & $N_{\mathrm{b}}$ & $\left(\mu_{x}, \mu_{y}\right)$ & $\left(-\mu_{x}, \mu_{y}\right)$ & $\left(-\mu_{x},-\mu_{y}\right)$ & $\left(\mu_{x},-\mu_{y}\right)$ & Cont. \\
\hline $12<B \leq 14$ & 173 & 28 & 4 & 3 & 4 & $14 \%$ \\
$14<B \leq 16$ & 1363 & 119 & 31 & 19 & 21 & $20 \%$ \\
$16<B \leq 18$ & 2321 & 228 & 68 & 50 & 51 & $25 \%$ \\
$18<B \leq 20$ & 1838 & 259 & 95 & 82 & 148 & $42 \%$ \\
\hline
\end{tabular}


Table 7. Candidate members of Stock 2 selected using CCD photometry and proper motions, sorted by $V$-magnitude. Stars that satisfy both the CCD photometric and proper motion selection criteria are labelled "Y" in the last column (22 stars). Five more stars which were judged as members from CCD photometry, possess proper motions that formally do not fulfill the selection criteria, but which are located close to members in the VPD diagram, and hence are judged to be possible members and are added with a "N?". In addition we include 13 of the CCD selected candidates for which is was not possible to derive proper motions, labelled with a "?", as they could be members

\begin{tabular}{|c|c|c|c|c|c|c|c|c|c|}
\hline \multirow{2}{*}{$\frac{\text { No. }}{1}$} & \multicolumn{2}{|c|}{$\alpha(\mathrm{J} 2000.0) \delta$} & \multirow{2}{*}{$\frac{V}{14.28}$} & \multirow{2}{*}{$\frac{B-V}{1.30}$} & \multirow{2}{*}{$\frac{V-R}{0.61}$} & \multirow{2}{*}{$\frac{R-I}{0.68}$} & \multirow{2}{*}{$\frac{\mu_{x}}{11.8}$} & \multirow{2}{*}{$\frac{\mu_{y}}{-22.9}$} & \multirow{2}{*}{$\begin{array}{l}\text { member? } \\
\mathrm{Y}\end{array}$} \\
\hline & $2: 14: 24.80$ & $59: 25: 39.1$ & & & & & & & \\
\hline 2 & $2: 14: 00.51$ & $59: 19: 55.8$ & 14.65 & 1.22 & 0.73 & 0.75 & - & - & $?$ \\
\hline 3 & $2: 15: 34.06$ & $59: 17: 05.6$ & 14.67 & 1.22 & 0.72 & 0.73 & 9.4 & -12.7 & $\mathrm{~N} ?$ \\
\hline 4 & $2: 15: 14.98$ & $59: 18: 51.5$ & 14.69 & 1.20 & 0.73 & 0.71 & 17.6 & -19.0 & $\mathrm{Y}$ \\
\hline 5 & $2: 13: 59.86$ & $59: 16: 45.6$ & 14.85 & 1.28 & 0.76 & 0.77 & 14.7 & -5.0 & $\mathrm{~N} ?$ \\
\hline 6 & $2: 15: 42.66$ & $59: 31: 23.4$ & 14.86 & 1.19 & 0.63 & 0.71 & - & - & $?$ \\
\hline 7 & $2: 15: 55.42$ & $59: 16: 28.7$ & 14.89 & 1.25 & 0.75 & 0.83 & - & - & $?$ \\
\hline 8 & $2: 13: 36.99$ & $59: 22: 54.5$ & 15.17 & 1.40 & 0.80 & 0.73 & 26.6 & -13.8 & $\mathrm{Y}$ \\
\hline 9 & $2: 15: 46.29$ & $59: 15: 25.0$ & 15.23 & 1.26 & 0.71 & 0.77 & 13.8 & -9.2 & $\mathrm{Y}$ \\
\hline 10 & $2: 15: 55.36$ & $59: 17: 45.5$ & 15.26 & 1.40 & 0.81 & 0.78 & 18.7 & -14.2 & $\mathrm{Y}$ \\
\hline 11 & $2: 15: 40.28$ & $59: 11: 34.6$ & 15.32 & 1.31 & 0.77 & 0.85 & - & - & $?$ \\
\hline 12 & $2: 16: 01.51$ & $59: 14: 06.9$ & 15.41 & 1.43 & 0.82 & 0.85 & 21.1 & -11.9 & $\mathrm{Y}$ \\
\hline 13 & $2: 15: 04.19$ & $59: 15: 43.3$ & 15.46 & 1.54 & 0.80 & 0.76 & 13.8 & -13.7 & $\mathrm{Y}$ \\
\hline 14 & $2: 14: 38.74$ & $59: 22: 16.3$ & 15.73 & 1.42 & 0.73 & 0.80 & 16.3 & -7.5 & $\mathrm{Y}$ \\
\hline 15 & $2: 14: 17.63$ & $59: 25: 12.0$ & 15.88 & 1.46 & 0.75 & 0.83 & 10.0 & -11.9 & $\mathrm{~N} ?$ \\
\hline 16 & $2: 14: 42.58$ & $59: 12: 48.9$ & 15.92 & 1.56 & 0.99 & 0.84 & - & - & $?$ \\
\hline 17 & $2: 14: 19.26$ & $59: 15: 53.7$ & 16.15 & 1.46 & 0.94 & 0.82 & - & - & $?$ \\
\hline 18 & $2: 14: 12.06$ & $59: 30: 22.1$ & 16.38 & 1.65 & 0.94 & 0.84 & 14.8 & -15.2 & $\mathrm{Y}$ \\
\hline 19 & 2:15:01.19 & $59: 08: 25.1$ & 16.46 & 1.75 & 1.13 & 1.10 & 12.8 & -12.9 & $\mathrm{Y}$ \\
\hline 20 & $2: 14: 58.08$ & 59:16:08.1 & 16.49 & 1.56 & 0.97 & 0.90 & 13.1 & -15.6 & $\mathrm{Y}$ \\
\hline 21 & $2: 15: 42.01$ & $59: 31: 26.7$ & 16.49 & 1.57 & 0.94 & 0.98 & - & - & $?$ \\
\hline 22 & $2: 13: 45.01$ & $59: 20: 43.9$ & 16.55 & 1.61 & 0.93 & 0.92 & 18.7 & -15.4 & $\mathrm{Y}$ \\
\hline 23 & $2: 15: 06.70$ & $59: 22: 37.9$ & 16.70 & 1.71 & 0.85 & 1.27 & 16.6 & -16.2 & $\mathrm{Y}$ \\
\hline 24 & $2: 14: 10.64$ & $59: 23: 40.5$ & 17.16 & 1.79 & 1.07 & 1.04 & 15.3 & -9.3 & $\mathrm{Y}$ \\
\hline 25 & $2: 15: 13.90$ & $59: 23: 51.6$ & 17.27 & 1.78 & 0.89 & 1.29 & 12.8 & -12.8 & $\mathrm{Y}$ \\
\hline 26 & $2: 15: 44.26$ & $59: 20: 50.0$ & 17.41 & 1.73 & 1.09 & 1.10 & - & - & $?$ \\
\hline 27 & $2: 15: 04.66$ & $59: 26: 22.7$ & 17.55 & 1.79 & 0.91 & 1.31 & 14.6 & -6.3 & $\mathrm{~N} ?$ \\
\hline 28 & $2: 14: 06.11$ & $59: 21: 31.1$ & 17.57 & 1.78 & 1.04 & 1.05 & 10.1 & -15.7 & $\mathrm{Y}$ \\
\hline 29 & $2: 14: 57.79$ & $59: 13: 07.8$ & 17.83 & 1.83 & 1.29 & 1.21 & - & - & $?$ \\
\hline 30 & $2: 15: 09.72$ & $59: 19: 19.3$ & 17.95 & 1.87 & 1.25 & 1.20 & 15.6 & -13.3 & $\mathrm{Y}$ \\
\hline 31 & $2: 13: 43.84$ & $59: 17: 28.0$ & 18.21 & 1.77 & 1.20 & 1.39 & 18.9 & -2.5 & $\mathrm{~N} ?$ \\
\hline 32 & $2: 14: 23.83$ & $59: 15: 37.2$ & 18.26 & 1.80 & 1.28 & 1.26 & 20.3 & -9.4 & $\mathrm{Y}$ \\
\hline 33 & $2: 14: 12.03$ & $59: 13: 26.9$ & 18.29 & 1.85 & 1.26 & 1.23 & - & - & $?$ \\
\hline 34 & $2: 13: 47.03$ & $59: 31: 37.2$ & 18.48 & 1.83 & 1.37 & 1.48 & 28.5 & -16.9 & $\mathrm{Y}$ \\
\hline 35 & $2: 13: 46.62$ & $59: 16: 03.7$ & 18.90 & 1.82 & 1.22 & 1.53 & - & - & $?$ \\
\hline 36 & $2: 13: 44.81$ & $59: 20: 09.2$ & 18.93 & 1.81 & 1.26 & 1.40 & 26.4 & -13.6 & $\mathrm{Y}$ \\
\hline 37 & $2: 14: 59.51$ & $59: 17: 05.3$ & 18.96 & 1.94 & 1.37 & 1.48 & - & - & $?$ \\
\hline 38 & $2: 13: 55.33$ & $59: 22: 18.1$ & 19.08 & 1.87 & 1.28 & 1.34 & 18.6 & -15.9 & $\mathrm{Y}$ \\
\hline 39 & $2: 14: 19.69$ & $59: 23: 57.6$ & 19.30 & 1.91 & 1.16 & 1.58 & 13.2 & -10.6 & $\mathrm{Y}$ \\
\hline 40 & $2: 15: 54.71$ & $59: 23: 20.0$ & 19.82 & 1.91 & 1.45 & 1.54 & - & - & $?$ \\
\hline
\end{tabular}

a lower membership probability are shown in the bottom two panels. Stars brighter than or equal to $B=16$ are plotted to the left, stars fainter than that to the right. This figure clearly shows the possible problem resulting from the lack of calibrating stars across the entire field of interest, namely, the distribution of photometrically selected stars is highly uneven, and is magnitude dependent. As was argued in Sect. 3.2, since it is uncertain to what extent this is a real effect as opposed to just incorrect calibration, no ad hoc changes to the photometric selection have been made.

Figure 5 also indicates that some of the structure seen in the distribution of the candidate members may be the result of the background structure. A clearer indication of the cluster can be seen when the positional distributions are converted into star densities, and the photometrically selected star density is subtracted from the candidate member density, as shown in Fig. 6. 

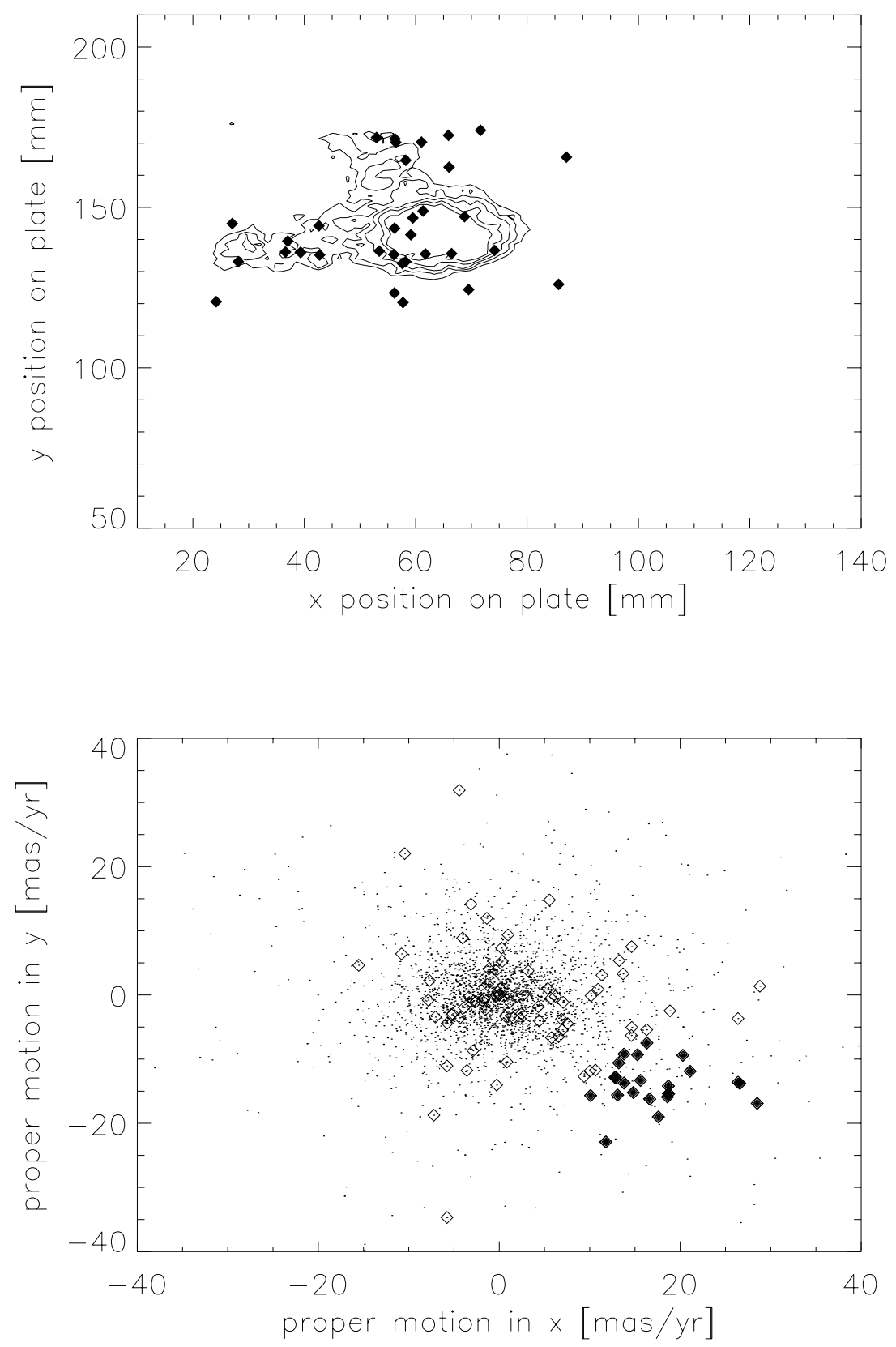

Fig. 6. The difference between the star density distributions of members and non-members. The diamonds indicate the position of previously determined cluster members taken from Krzemiński \& Serkowski (1967)

Fig. 7. Proper motions for stars with CCD photometry. Diamonds denote those stars which fulfill both selection criteria in the $(B, B-V)$ and $(R, R-I)$ CMDs. Filled symbols represent those stars with a membership probability greater than $50 \%$ based on the proper motion membership criteria. There are 22 such stars (see Table 7), but only 21 appear in this plot because two stars have almost identical proper motions
The cluster is clearly visible as a density enhancement. Given that the plate scale is $67.14 \operatorname{arcsec} / \mathrm{mm}$, this would imply that the cluster has a spatial diameter of approximately 30 arcmin. This is much smaller than the value of 60 arcmin quoted by Lynga (1987) based on the distribution of early-type cluster members. Figure 6 also shows the positions of 34 stars previously determined to be cluster members by Krzemiński \& Serkowski (1967). These positions agree with our density distribution. The small diameter of the peak in the density may be indicative of the cluster core rather than the entire cluster. Certainly, the list of stars with membership probabilities less than 50\% contains some cluster members, and so the spatial density of the "non-members" will be enhanced, which may explain why only the cluster core is evident.

\subsection{Analysis of members selected from CCD photometry}

The proper motions of all the stars with CCD photometry are plotted in Fig. 7, showing the positions of the candidate members as open diamonds. Combining the results of the proper motion study, we find 22 stars with membership probabilities greater than $50 \%$. This would imply that the 
list of candidate members as based on CCD photometry alone is contaminated by up to $80 \%$ with non-members. The level of contamination in the sample can be estimated from the number of stars located at a similar distance from the origin in a different quadrant of the plot. It is clear that the final candidate list is likely to contain at most one or two non-members.

Figure 7 shows one of the problems with the determination of the membership probabilities as discussed in the previous section. There are roughly 6 stars with proper motions that lie close in the VPD to the sharp cut-off boundary illustrated in Fig. 3. These stars have membership probabilities that are slightly less than $50 \%$ and hence have been formally deemed "non-members" in the proper motion selection process. For this reason, these stars are listed in Table 7 with membership "N?".

\section{Conclusions}

$B V R I$ CCD photometry has been obtained for $215 \times$ 5 arcmin fields in the region of Stock 2. From a photometric selection using two colour-magnitude diagrams, 118 candidate cluster members have been identified. These range from $V=14.2$ to $V=20.4$, corresponding to spectral type from mid-K to late-M. We compared these results with the observed luminosity and mass-distribution functions for the Pleiades (Hambly \& Jameson 1991). After scaling the distance modulus of the Pleiades to Stock 2, our CCD study would have resulted in the detection of 52 Pleiads. Since we found 118 Stock 2 member candidates, the contamination of this sample was estimated to be roughly $50 \%$. This comparison is justified by Lynga's (1987) classification of "richness index" for both clusters. However, the richness index is a rough classification based on the observed number of early-type stars in clusters, and the mass-distribution of late-type stars may be different.

The results of a proper motion survey of Stock 2 are also presented. The survey covered an area of $3^{\circ} \times 3^{\circ}$ about the nominal cluster centre. The selection of candidate cluster members was based on an initial selection from a photographically determined colour-magnitude diagram. The analysis identified 634 stars with magnitudes from $B=12.8$ to $B \sim 20$. The contamination of the sample was estimated to range from $<20 \%$ in the brightest bin to $>40 \%$ in the faintest bin.

The results of the proper motion analysis were also applied to the CCD determined candidate members. Of the 118 selected stars, 22 have proper motions consistent with cluster membership, with perhaps only one or two field stars present in the list. This is less than half of the expected number of cluster members in the area covered by the CCD photometry, estimated from comparison with the Pleiades. This suggests that while both clusters have the same "richness index", the core of Stock 2 contains fewer stars than the core of the Pleiades.

Acknowledgements. We gratefully acknowledge the help and assistance of Nigel Hambly in reducing and analysing the proper motion data. Data reduction was performed on the PPARC funded Northern Ireland STARLINK node. DCF acknowledges support of a studentship from Armagh observatory. Research at Armagh Observatory is funded by a grantin-aid from DENI. AT acknowledges support from PPARC grants GR/J25352, GR/L21594 and GR/L29859. WRJR also acknowledges financial assistence from the PPARC. SLH acknowledges partial support from NSF grant \# AST94-57455.

\section{References}

Alexander D.R., Augason G.C., Johnson H.R., 1989, ApJ 345, 1014

Barrado D., Byrne P.B., 1995, A\&AS 111, 275

Beard S.M., MacGillivray H.T., Thanisch P.F., 1990, MNRAS 247,311

Bessell M.S., 1986, PASP 98, 1303

Bessell M.S., 1991, AJ 101, 662

Bessell M.S., 1995, in: The Bottom of the Main Sequence - and Beyond Tinney C. (ed.). Springer, Heidelberg, p. 123

Blair M., Filmore G., 1982, PASP 94, 742

Canuto V.M., Mazzitelli I., 1991, ApJ 370, 295

Dabrowski J.P., Beardsley W.R., 1977, PASP 89, 225

D'Antona F., Mazzitelli I., 1994, ApJS 90, 467

Foster D.C., Byrne P.B., Hawley S.L., Rolleston W.R.J., 1997, A\&AS 126,81

Hambly N.C., Jameson R.F., 1991, MNRAS 249, 137

Hambly N.C., Steele I.A., Hawkins M.R.S., Jameson R.F., 1995, A\&AS 109, 29

Hambly N.C., Miller L., MacGillivray H.T., Herd J.T., Cormack W.A., 1998, MNRAS 298, 897

Jeffries R.D., 1997, MNRAS 292, 177

Jeffries R.D., Tolley A.J., 1998, MNRAS 300, 331

Jones B.F., Stauffer J.R., 1991, AJ 102, 1080

Kirkpatrick J.D., Kelly D.M., Rieke G.H., et al., 1993, ApJ 402,643

Krzemiński W., Serkowski K., 1967, ApJ 147, 988

Landolt A.U., 1992, AJ 104, 340

Lynga G., 1987, in Catalogue of Open Cluster Data, Strasbourg

Pandey A.K., Bhatt B.C., Mahra H.S., Sagar R., 1989, MNRAS 236, 263

Piskunov A., 1980, Bull. Inf. Cent. Données Stellaires 19, 67

Press W.H., Teukolsky S.A., Vitterling W.T., Flannery B.P., 1992, Numerical Recipes in Fortran. Cambridge University Press

Prosser C.F., Stauffer J.R., Caillault J.-P., Balachandran S., Stern R.A., Randich S., 1995, AJ 110, 1229 
Randich S., Schmitt J.H.M.M., Prosser C.F., Stauffer J.R., 1995, A\&A 300, 134

Rodgers F.J., Iglesias C.A., 1992, ApJS 79, 507

Robichon N., Arenou F., Turon C., Mermilliod J.-C., Lebreton Y., 1997, in: Proceedings of the ESA Symposium "Hipparchos - Venice '97", ESA SP-402, p. 567

Robichon N., Arenou F., Mermilliod J.-C., Turon C., 1999, A\&A 345, 471

Rolleston W.R.J., Byrne P.B., 1997, A\&AS 126, 357

Sanders W.L., 1971, A\&A 14, 226
Stauffer J.R., Hartmann L.W., Barrado y Navascues D., 1995, ApJ 454, 910

Stauffer J.R., Hartmann L.W., Prosser C.F., et al., 1997, ApJ 479,776

Stetson P.B., Davis L.E., Crabtree D.R., 1990, Future development of the DAOPHOT crowded-field photometry package, in: CCDs in Astronomy, ASP Conf. Ser. 8, 289

Stetson P.B., 1987, PASP 99, 191

Stock J., 1956, ApJ 123, 258

Tody D., 1986, in IRAF User Manual 